\title{
Nitazoxanida: aspectos gerais, sistemas de liberação e potencial de reposicionamento
}

\section{da molécula}

\author{
Nitazoxanide: general aspects, release systems and potential for repositioning the molecule \\ Nitazoxanida: aspectos generales, sistemas de liberación y potencial de reposicionamiento de la \\ molécula
}

\section{Resumo}

A nitazoxanida (NTZ) é um fármaco empregado no tratamento de infecções virais e parasitárias devido ao seu mecanismo de ação ativar processos de morte celular. Entretanto, apresenta baixa biodisponibilidade oral, restringindo a sua utilização na terapêutica, com isso, novos sistemas de liberação são necessários para melhor absorção in vivo. O objetivo desta revisão narrativa foi abordar aspectos referentes a características físico-químicas da molécula de Nitazoxanida e seu potencial de reposicionamento. Foram realizadas buscas nas bases de dados Pubmed, Lilacs e Science Direct, selecionados artigos em inglês e português, cruzando os descritores "Nitazoxanida", "caracterização" e "formulação" combinados entre si. Evidências científicas descrevem a NTZ como uma molécula de baixo peso molecular, melhor absorvida em pH ácido, que sofre desacetilação originando seu metabólito ativo, a Tizoxanida. Estudos relatam sua aplicação frequentemente em doenças por Giardia lamblia, Entamoeba histolytica e Cryptosporidium parvum, além de infecções por protozoários, helmintos, bactérias gram negativas e positivas e possuir propriedades antivirais, estando relacionado a sua descoberta recente na COVID-19, inclusive associada a outros fármacos. Assim, sistemas como lipossomas, nanopartículas, microesferas, comprimidos de liberação controlada foram relatados como potencializadores veículos na liberação do fármaco, além de possibilitar melhoria nos seus aspectos físico-químicos. Portanto, a NTZ representa uma molécula com grande potencial de aplicação e reposicionamento, abrangendo inúmeras possibilidades.

Palavras-chave: Formulação; Antiparasitários; Farmacologia; Nitazoxanida; Tizoxanida.

\begin{abstract}
Nitazoxanide (NTZ) is a drug used in the treatment of viral and parasitic infections due to its mechanism of action to activate cell death processes. However, it has low oral bioavailability, restricting its use in therapy, with this, new delivery systems are necessary for better absorption in vivo. The objective of this narrative review was to address aspects related to the physicochemical characteristics of the Nitazoxanide molecule and its potential for repositioning. Searches were carried out in the Pubmed, Lilacs and Science Direct databases, selecting articles in English and Portuguese, crossing the descriptors "Nitazoxanida", "characterization" and "formulation" combined. Scientific evidence describes NTZ as a low molecular weight molecule, better absorbed at acidic $\mathrm{pH}$, which undergoes deacetylation giving rise to its active metabolite, Tizoxanide. Studies report its application frequently in diseases by Giardia lamblia, Entamoeba histolytica and Cryptosporidium parvum, in addition to infections by protozoa, helminths, gram negative and positive bacteria and having antiviral properties, being related to its recent discovery in COVID-19, even associated with others drugs. Thus, systems such as liposomes, nanoparticles, microspheres, controlled-release tablets have been reported as vehicle enhancers in the release of the drug, in addition to enabling improvement in its physical-chemical aspects. Therefore, NTZ represents a molecule with great potential for application and repositioning, covering numerous possibilities.
\end{abstract}

Keywords: Formulation; Antiparasitic; Pharmacology; Nitazoxanide; Tizoxanide. 


\begin{abstract}
Resumen
La nitazoxanida (NTZ) es un fármaco utilizado en el tratamiento de infecciones virales y parasitarias debido a su mecanismo de acción para activar los procesos de muerte celular. Sin embargo, tiene baja biodisponibilidad oral, lo que restringe su uso en terapia, por lo que son necesarios nuevos sistemas de administración para una mejor absorción in vivo. El objetivo de esta revisión narrativa fue abordar aspectos relacionados con las características fisicoquímicas de la molécula de Nitazoxanida y su potencial de reposicionamiento. Las búsquedas se realizaron en las bases de datos Pubmed, Lilacs y Science Direct, seleccionando artículos en inglés y portugués, cruzando los descriptores "Nitazoxanida", "caracterización" y "formulación" combinados. La evidencia científica describe al NTZ como una molécula de bajo peso molecular, mejor absorbida a pH ácido, que sufre desacetilación dando lugar a su metabolito activo, la tizoxanida. Los estudios reportan su aplicación frecuentemente en enfermedades por Giardia lamblia, Entamoeba histolytica y Cryptosporidium parvum, además de infecciones por protozoos, helmintos, bacterias gramnegativas y positivas y que tienen propiedades antivirales, estando relacionado con su reciente descubrimiento en COVID-19, incluso asociado con otras Drogas. Así, sistemas como liposomas, nanopartículas, microesferas, comprimidos de liberación controlada se han reportado como vehículos potenciadores en la liberación del fármaco, además de permitir la mejora en sus aspectos físicoquímicos. Por tanto, NTZ representa una molécula con gran potencial de aplicación y reposicionamiento, cubriendo numerosas posibilidades.
\end{abstract}

Palabras clave: Formulación; Antiparasitario; Farmacología; Nitazoxanida; Tizoxanida.

\title{
1. Introdução
}

A nitazoxanida (NTZ) e seu metabólito ativo tizoxanida (TIZ) são compostos pertencentes ao grupo das tiazolidas, com amplo espectro de propriedades farmacológicas (Padmanabhan, 2020) e a literatura relata que em humanos é eficaz contra parasitas como Giardia lamblia, Entamoeba histolytica e Cryptosporidium Parvum (Abaza, El-Zayadi, Kabil, \& Rizk, 1998). Assim, de acordo com Rossignol (2014); Rossignol (2016) e Shalan, Nasr e Belal (2014), a Nitazoxanida (NTZ) [2 - [(5-nitro1,3-tiazol-2-il) carbamoil] fenil] acetato, é um derivado de nitrotiazol sintético com atividade contra infecções por protozoários, helmintos, bactérias gram negativas e gram positivas e diversos vírus (vírus respiratórios, rotavírus, norovírus, coronavírus, hepatite B e C, dengue-2, febre amarela, encefalite japonesa e vírus da imunodeficiência humana).

Dessa forma, a estrutura da molécula de NTZ é formada por dois grupamentos: uma porção nitrotiazol e uma porção de ácido salić́lico interligada por uma ligação amida. Nesse sentido, o grupamento nitro e a porção nitrotiazol podem ser convertidas em radical livre desencadeando eventos de morte celular por interferência em vias de sinalização e são consideradas detentoras da função contra parasitas extracelulares. Além disso, estudos apontam que a porção nitro está envolvida na inibição da piruvatoferredoxina oxidorredutase (PFOR), enzima essencial para o metabolismo de organismos anaeróbicos (Fan-Minogue et al., 2013).

Por outro lado, sugere-se que a atividade antiviral esteja relacionada com ativação da proteína quinase ativada por RNA de cadeia dupla, induzindo a síntese de interferon que medeiam a imunidade celular antiviral (Shigyo et al., 2016). A eficácia antitumoral é atribuída a diferentes mecanismos envolvendo autofagia, atividades anticitocinas, inibição de c-Myc (Fan-Minogue et al., 2013) e supressão da produção de interleucinas (Hong et al., 2012), sendo um composto promissor destacado para o tratamento da dor neuropática e do vírus Ebola (Jasenosky et al., 2019; Ai, Wood \& Welsh, 2015).

Devido à sua segurança e atividade comprovada em infecções virais e parasitárias, a NTZ está atualmente sob avaliação em ensaios clínicos para várias doenças. Diversos estudos demonstraram atividade contra Mycobaterium tuberculosis (Mishra et al., 2020), larvas de Taenia solium (Lima, Picanço, Costa, Junior, \& Vinaud, 2020), carcinoma de glândula mamária em ratos (Pal, Nandave, \& Kaithwas, 2020), toxoplasmose (Farahatallam et al., 2020) e mais recentemente a possibilidade do seu uso na COVID-19 (Kelleni, 2020), dentre outras múltiplas aplicações. 
De acordo com Malesuik, Paim, Schapoval e Steppe (2010), a nitazoxanida tem baixa solubilidade aquosa, sendo considerada praticamente insolúvel, o que torna uma característica significante de sua molécula. Assim, existe a necessidade da busca pelo desenvolvimento de novas estratégias farmacêuticas de liberação e veiculação que melhorem a sua biodisponibilidade, visando sua estabilidade e fornecendo uma terapêutica mais eficaz, assim como a oportunidade de potencializar sua aplicação em diversos tipos de morbidades.

Desse modo, o objetivo desta revisão narrativa foi abordar os aspectos referentes as características físico-químicas da molécula de Nitazoxanida e sua aplicação no reposicionamento de fármacos.

\section{Metodologia}

O presente estudo trata-se de uma revisão bibliográfica narrativa acerca da Nitazoxanida, no qual foram levantadas e discutidas informações sobre os aspectos gerais da molécula assim como estudos de desenvolvimento de novas formulações.

Os artigos de revisão narrativa são publicações apropriadas para descrever e discutir o desenvolvimento de um determinado assunto sob o ponto de vista teórico ou contextual, evidenciando novas ideias, métodos, subtemas que têm recebido maior ou menor ênfase na literatura selecionada (Noronha \& Ferreira, 2000; Rother, 2007). Esse tipo de estudo tem papel fundamental para a educação continuada, pois permite ao leitor adquirir e atualizar o conhecimento sobre um tema específico em um curto espaço de tempo (Sallum, Garcia, \& Sanches, 2012; Elias et al., 2012).

Para a elaboração da revisão narrativa compilou-se artigos durante o mês de agosto a dezembro de 2020, utilizando as bases eletrônicas Lilacs, Science Direct e PubMed, com os seguintes descritores: "nitazoxanide", e quando necessário "and" "characterization" e "and" "formulation", que foram combinados entre si para aprimorar a seleção dos artigos. Além disso, foram excluídos artigos que muito antigos, que não abrangiam o foco do estudo e não apresentavam uma possibilidade para reposicionamento da molécula.

\section{Propriedades Físico-Químicas da Molécula}

A Nitazoxanida (NTZ) foi descrita pela primeira vez em 1984 como um fármaco cestocida humano que foi eficaz em dose única contra Taenia saginata e Hymenolepis nana (Rossignol \& Maisonneuve, 1984). A estrutura da molécula é mostrada na figura 1.

Figura 1. Estrutura química da molécula de NTZ

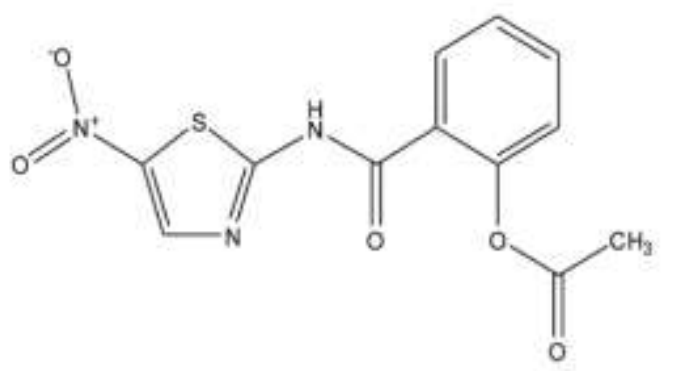

Fonte: Malesuik (2012).

A NTZ apresenta formula molecular $\mathrm{C}_{12} \mathrm{H}_{9} \mathrm{~N}_{3} \mathrm{O}_{5} \mathrm{~S}$, peso molecular de $307,28 \mathrm{~g} / \mathrm{mol}$, classe II (Baixa solubilidade e alta permeabilidade) no Sistema de Classificação Biofarmacêutica e faixa de pH que garante a estabilidade entre 1-4. Por outro lado, situações extremas de pH, 0,01 e 10, levam a maior degradação do fármaco. Demonstrou ser instável na forma sólida e em 
solução por diversos fatores, como: calor, oxidação, ácido, básico e luz. Em estudos de estabilidade foram testadas experimentalmente várias condições que afetassem esses parâmetros como decomposição térmica, fotólise, condições ácidas, alcalinas e oxidativas. Como resultado obtido observou-se níveis diferentes de decomposição quando a NTZ foi submetida a essas condições. A decomposição térmica diminuiu a porcentagem do fármaco nas amostras em 20\%, a fotólise 10\% após 15 minutos, condições ácidas reduziu $70 \%$ da concentração e alcalinas $60 \%$ após $2 \mathrm{~h}$ e a reação oxidativa causou degradação total do fármaco após 30 minutos. A degradação da NTZ nas condições estudadas seguiu uma cinética de reação de primeira ordem (Malesuik, Gonçalves, Garcia, Trein, Nardi, Schapoval, \& Steppe, 2012).

Em relação às suas características de absorção, em humanos, a NTZ é parcialmente absorvida no trato gastrointestinal, com aproximadamente um terço da dose oral excretada na urina e dois terços nas fezes. No sangue, a NTZ é metabolizada para formar um metabólito ativo, a tizoxanida, seguido pela formação de conjugados glucuronídeos e sulfônicos (Rossignol \& Stachulski, 1999; Broekhuysen, Stockis, Lins, De Graeve, \& Rossignol, 2000).

\section{Nitazoxanida: atividades farmacológicas}

Nitazoxanida [2 - [(5-nitro-1,3-tiazol-2-il) carbamoil] fenil] acetato é um composto antiparasitário de amplo espectro pertencente a uma classe nitroheterocíclica denominada tiazolida com atividade contra protozoários, nematódeos, cestódeos e trematódeos. Em humanos, a nitazoxanida é rapidamente metabolizada em tizoxanida, um composto tão eficaz quanto o fármaco original (White, 2004). É bem tolerada, tem poucos efeitos colaterais e requer um curto período de tratamento. Não há contraindicações importantes, exceto hipersensibilidade prévia (Gurgen, Hogan, Grace, \& Johnson, 2011). No entanto, ela requer monitoramento quando administrada concomitantemente com medicamentos como varfarina ou fenitoína e não há dados sobre a biodisponibilidade cutânea (Zhang et al., 2010).

Ademais, a nitazoxanida, e sua forma reduzida tizoxanida são eficazes contra a maioria dos patógenos algo, sendo o único medicamento que apresenta bons resultados em infecções por Cryptosporidium e Giardia lamblia (Miyamoto \& Eckmann, 2015) (Abaza et al, 1998). Soma-se a isso o fato de ser indicada contra infecções por helmintos intestinais e de tecidos, como equinococose cística (Laura et al., 2015), helmintos transmitidos pelo solo (Somvanshi, Ellis, Hu, \& Aroian, 2014), como Ancylostoma ceylanicum, Ascaris suum, Trichuris muris e Caenorhabditis elegans (Hu et al., 2013), Taenia saginata e Hymenolepis nana (Rossignol \& Maisonneuve, 1984). Além disso, a atividade da NTZ como tratamento para a doença por Clostridium difficile foi confirmada por estudos em animais e também por ensaios clínicos (Rossignol, 2014).

Kohla et al. (2016) aponta o uso da NTZ em doenças hepáticas, em seu estudo eles demonstraram o efeito da nitazoxanida na inibição da replicação do vírus da hepatite $\mathrm{C}$, avaliando o impacto da nitazoxanida como uma terapia complementar ao interferon peguilado $\alpha$-2a e ribavirina na resposta virológica sustentada (RVS) em pacientes com hepatite C crônica. Um total de 195 pacientes foram avaliados, entretanto como resultado encontrado o estudo não mostrou um impacto significativo da nitazoxanida na RVS.

Dhawan et al., 2015, descreve a NTZ como um tratamento eficaz da leishmaniose cutânea por Leishmania donovani (leishmaniose visceral). Esse estudo demonstra que a concentração de $200 \mu \mathrm{g} / \mathrm{mL}$ inibe o crescimento de $>90 \%$ dos promastigotas, mostrando atividade semelhante à do medicamento de referência Anfotericina B ( p > 0,05) (Zhang et al, 2010).

A nitazoxanida é um antiviral que já foi testado em ensaios clínicos de Fase III para o tratamento da gripe (Rossignol, 2014) e produz esse efeito bloqueando a maturação da glicoproteína viral HA (Rossignol, La Frazia, Chiappa, Ciucci, \& Santoro, 2009). Da mesma forma, Tilmanis e colaboradores (2020) investigando sobre a propensão dos vírus de influenza de desenvolver resistência revela que o uso de nitazoxanida como um antiviral para gripe é altamente improvável de resultar no surgimento de vírus resistentes, embora tenha demonstrado que a presença do fármaco não previne o surgimento e seleção de vírus resistentes ao oseltamivir. O mesmo autor em um estudo de 2017 demonstra o potente efeito antiviral de NTZ contra 210 vírus da gripe 
sazonal (Tilmanis et al, 2017). Um efeito antiviral sinérgico foi observado ao combinar NTZ com oseltamivir ou zanamivir contra influenza A/H1N1-PR8 e o aviário A/H5N9 (Belardo, Cenciarelli, La Frazia, Rossignol, \& Santoro, 2015).

Em um ensaio clínico humano desenvolvido por Haffizulla e colaboradores (2014), os autores mostraram que a NTZ pode reduzir efetivamente a duração dos sintomas da gripe em pacientes que receberam doses orais de $600 \mathrm{mg}$ duas vezes ao dia em comparação com o placebo. Ademais, não foi relatada resistência antiviral contra NTZ entre os vírus influenza tratados e nenhum evento adverso foi relatado no sistema imunológico humoral dos pacientes que receberam a droga. Também foi relatado que a NTZ impede a replicação de duas cepas de rotavírus (WaG1P (8) e o símio SA11-G3P (2)) (Rossignol, 2014).

Jasenosky et al. (2019) relataram recentemente que a NTZ poderia efetivamente amplificar a resposta imune inata do hospedeiro e inibir a replicação do vírus Ebola. O modo de ação descrito envolve o aumento do receptor de proteína I induzível por ácido retinóico, bem como a proteína de sinalização antiviral mitocondrial. Além disso, a NTZ estimula a expressão do fator regulador do interferon 3 e estimula a transcrição da fosfatase antiviral GADD34, apresentando assim uma abordagem promissora como terapia para a doença

Estudos abordando a relação de NTZ com chikungunya também foram encontrados e resultados mostrados por Weaver e Forrester (2015) apontam que a NTZ pode limitar a entrada e a liberação do vírus, bem como a transmissão de célula para célula. Além disso, o fármaco exibiu ampla atividade antiviral contra dois isolados clínicos do vírus chikungunya, bem como dois tipos de alfavírus (vírus Sindbis e o vírus da floresta Semliki) (Wang et al., 2016). Ademais, NTZ pode inibir efetivamente a replicação de do vírus dengue- 2 e febre amarela em linhas de células Vero com metade das concentrações inibitórias máximas de 0,1 e $0,06 \mu \mathrm{g} / \mathrm{ml}$, respectivamente (Botta, Rivara, Zuliani, \& Radi, 2018).

\section{Reposicionamento da Molécula: Nitazoxanida e Covid-19}

Existe um surto mundial de um novo tipo de coronavírus (COVID-19), que se originou em Wuhan, China, e já se espalhou para outros 140 países, incluindo Japão, Coréia e Itália. A Organização Mundial da Saúde (OMS) declarou que o COVID-19 se tornou um problema de saúde global, causando infecções graves do trato respiratório em humanos (Zhu et al, 2020; Lu et al, 2020). Os sintomas típicos da COVID-19 são febre, dor de garganta, fadiga, tosse ou dispneia associada à exposição recente (Harcourt et al, 2020). Em 30 de junho de 2020, a Organização Mundial da Saúde (OMS) registrou mais de 10 milhões de casos de COVID-19 com quase meio milhão de mortes em todo o mundo (Who, 2020).

Anteriormente, mostrou-se que a Nitazoxanida tem potencial antiviral, e estudos apontam o seu uso no tratamento do COVID-19. Em estudo proposto por Pepperrell, Pilkington, Owen, Wang e Hill (2020), demonstra-se que, ao contrário de outros fármacos postulados, a nitazoxanida mostra uma alta proporção da concentração plasmática máxima $\left(\mathrm{C}_{\max }\right)$, após 1 dia de 500 mg duas vezes ao dia (BD), para a concentração necessária para inibir a replicação de $50 \%$ (EC 50 ) da síndrome respiratória aguda grave coronavírus 2 ( SARS-CoV-2).

Este artigo estuda uma revisão da pesquisa clínica sobre nitazoxanida, acerca de ensaios clínicos randomizados de fase 2 ou 3. Desse modo, mostram que a nitazoxanida demonstra um bom perfil de segurança em doses aprovadas. No entanto, são necessárias mais evidências em relação aos efeitos hepatorrenais e cardiovasculares, bem como à teratogenicidade, além de outros ensaios em pacientes com COVID-19. Assim, demonstram que a nitazoxanida pode representar um tratamento seguro e acessível na pandemia em andamento (Pepperrell et al, 2020).

Em 2020, Wang e colaboradores relataram que o NTZ pode inibir SARS-CoV-2 em células Vero E6 (ATCC-1586) após infecção com SARS-CoV-2 / Wuhan / WIV04 / 20192 em uma concentração micromolar baixa (Wang et al, 2020).

Recentemente, Kelleni (2020) sugeriu o uso de NTZ com azitromicina como um protocolo para os casos iniciais de COVID-19, com base em uma abordagem fisiopatológica e farmacológica combinada. Ao final, Kelleni (2020) explicita que a combinação de nitazoxanida / azitromicina pode eventualmente parecer um regime mais seguro e eficaz. 
Atualmente, existem 14 ensaios clínicos para o uso de NTZ sozinho ou em combinação com outros medicamentos (ivermectina ou hidroxicloroquina) para tratar pacientes com COVID-19 (Mahmoud, Shitu, \& Mostafa, 2020). Os países que iniciaram os ensaios clínicos para NTZ são Egito, Estados Unidos, Brasil e México (Broekhuysen et al, 2000). Recentemente, no final do mês de dezembro, um estudo feito por Rocco et al. (2020) apresentou os dados do ensaio multicêntrico, randomizado, duplo-cego e controlado por placebo sobre o uso do NTZ no início da doença de Covid-19 em pacientes com sintomas leves: tosse seca, febre e/ou fadiga. Pacientes confirmados com SARS-CoV-2 foram divididos e um grupo recebeu NTZ $500 \mathrm{mg}$, enquanto o outro recebeu placebo, por cinco dias. Após a investigação, constatou-se que não houve diferença na resolução dos sintomas, entretanto houve uma redução da carga viral nos pacientes tratados com NTZ (55\%) ao iniciar o tratamento no início dos sintomas, em relação a redução com o placebo (45\%), mostrando que a além da ação na redução da carga viral, o NTZ se apresentou como um fármaco seguro.

Ademais, o NTZ representa um medicamento promissor para o ensaio clínico contra COVID-19 devido à sua capacidade de controlar as respostas imunes inflamatórias excessivas, seu efeito broncodilatador e atividade anti-SARS-CoV-2 in vitro (Mahmoud, Shitu, \& Mostafa, 2020). Entretanto, novos estudos clínicos com seu uso isolado e/ou associado se faz necessário para comprovação da sua efetiva utilização na prática clínica.

\section{Sistemas de Liberação de Fármacos: novas formulações}

Estudos relatados em revisão por Gilles \& Hoffman, 2002, explicita o uso de NTZ em pacientes sintomáticos, seguindo protocolos elaborados de acordo com as diretrizes para a avaliação de medicamentos para giardíase e tratamento da amebíase, no qual foi administrado na dose de $500 \mathrm{mg}$ a cada 12 horas por três dias em adultos e adolescentes, $200 \mathrm{mg}$ a cada 12 horas por três dias em crianças de 4 a 11 anos e $100 \mathrm{mg}$ a cada 12 horas por três dias em crianças de 1-3 anos. Entretanto, tendo em vista o seu uso atual e devido à baixa solubilidade aquosa, o NTZ tem baixa biodisponibilidade e requer altas doses para o tratamento (Félix-Sonda, Rivera-Islas, Herrera-Ruiz, Morales-Rojas, \& Höpfl, 2014). Matysiak-Budnik, 2002, o configura como possuidor de elevada permeabilidade através do epitélio intestinal, sendo, assim, um medicamento classe II de acordo com os critérios estabelecidos pelo Sistema de Classificação Biofarmacêutica (BCS).

Para aproveitar o amplo espectro terapêutico do NTZ, é necessária a implementação de estratégias para modificar sua solubilidade. Uma estratégia para superar a solubilidade limitada de fármacos com dificuldade de liberação ou absorção é o desenvolvimento de dispersões de fases sólidas (Duggirala, 2016; Bolla \& Nangia, 2016; Wouters \& Quéré, 2012).

Os dados referentes à busca por publicações na literatura sobre sistemas de liberação com NTZ estão apresentados na Tabela 1.

Tabela 1: Relação de autores selecionados, formulações e ensaios de caracterização descritas na literatura.

\begin{tabular}{lcc}
\hline Autor & Sistema de Liberação & Ensaios de caracterização \\
\hline $\begin{array}{c}\text { Darwish, Bayoumi e El-Kolaly, } \\
2018\end{array}$ & Lipossoma & $\begin{array}{c}\text { Microscopia eletrônica de varredura, } \\
\text { Espalhamento Dinâmico de Luz (DLS) }\end{array}$ \\
\hline Sanopartícula & Cocristais & $\begin{array}{c}\text { Análise de difração de raios-X de pó (PXRD), } \\
\text { Análise de Microscopia Eletrônica de } \\
\text { Varredura (MEV) }\end{array}$ \\
\hline
\end{tabular}




\begin{tabular}{|c|c|c|}
\hline \multirow[t]{4}{*}{$\begin{array}{l}\text { Sachan, Gupta, Kumari e Ansari, } \\
2018\end{array}$} & Microesferas & $\begin{array}{c}\text { Tamanho de partícula, teor do fármaco, } \\
\text { Avaliação Morfológica, }\end{array}$ \\
\hline & & $\begin{array}{l}\text { Difratometria de raios-X em pó (XRD), DSC, } \\
\text { PXRD, }\end{array}$ \\
\hline & & Densidade aparente \\
\hline & & Densidade testada \\
\hline \multirow[t]{3}{*}{ Suresh et al., 2017} & $\begin{array}{c}\text { Comprimidos de liberação } \\
\text { prolongada }\end{array}$ & $\begin{array}{l}\text { Variação de peso, espessura, friabilidade e } \\
\text { teor do fármaco, Ângulo de repouso: }\end{array}$ \\
\hline & & Densidade aparente \\
\hline & & Densidade testada, teor do fármaco \\
\hline
\end{tabular}

\begin{tabular}{ccc}
\hline $\begin{array}{c}\text { Abbasalipourkabir,Fallah, } \\
\text { Sedighi, Maghsood e Javid, 2016 }\end{array}$ & Nanopartículas lipídicas sólidas & $\begin{array}{c}\text { Tamanho de partícula, potencial zeta, índice } \\
\text { de polidispersão, DSC, FTIR, eficiência de } \\
\text { encapsulamento }\end{array}$ \\
\hline
\end{tabular}

Srikala, Priya e Nadendla, 2020

Srikala, Priya e Nadendla, 2020
Comprimidos com hidrogel superporoso

Uniformidade de peso, dureza, friabilidade, espessura, uniformidade de conteúdo, Ângulo de repouso, Densidade aparente

Densidade testada, medição de porosidade, fator de Hausner, Índice de Carr

Calorimetria exploratória diferencial (DSC), Espectroscopia Infravermelho, espectroscopia UV, dureza, variação de peso, espessura, friabilidade e teor do fármaco

Mohan e Alur, 2019

Comprimidos de liberação sustentada

Teor do fármaco, Índice de Carr, Densidade derivada, Densidade aparente, Ângulo de repouso, Dureza,

Espessura, peso, friabilidade, doseamento do fármaco por HPLC.

\begin{tabular}{|c|c|c|}
\hline $\begin{array}{c}\text { Golamaru, Rajnarayana e } \\
\text { Jayaveera, } 2016\end{array}$ & $\begin{array}{l}\text { Comprimidos de liberação } \\
\text { sustentada }\end{array}$ & $\begin{array}{l}\text { Densidade aparente e compactada, índice de } \\
\text { Carr, fator de Hausner, ângulo de repouso, } \\
\text { espessura, dureza, friabilidade, uniformidade } \\
\text { de unidades de dosagem, doseamento do } \\
\text { fármaco por HPLC }\end{array}$ \\
\hline $\begin{array}{c}\text { Ahirrao, Rathi, Koli, Kshirsagar e } \\
\text { Pawar, } 2018\end{array}$ & Comprimidos orodispersíveis & $\begin{array}{l}\text { Solubilidade, teor do fármaco, espectroscopia } \\
\text { infravermelha, calorimetria de varredura } \\
\text { diferencial, difração de raios-X, dissolução, } \\
\text { ângulo de repouso, densidade, índice de Carr, } \\
\text { uniformidade de peso e conteúdo, dureza, } \\
\text { friabilidade, umedecimento, desintegração. }\end{array}$ \\
\hline $\begin{array}{c}\text { Félix-Sonda, Rivera-Islas, } \\
\text { Herrera-Ruiz, Morales-Rojas, \& } \\
\text { Höpfl, } 2014\end{array}$ & $\begin{array}{c}\text { Cocristais com ácido succinico e } \\
\text { ácido glutárico }\end{array}$ & $\begin{array}{l}\text { Espectroscopia IV, espectroscopia UV-vis, } \\
\text { análise termogravimétrica, calorimetria de } \\
\text { varredura diferencial, análises de difração de } \\
\text { raios-X em pó, cristal único, ensaios } \\
\text { biofarmacêuticos ( teste de estabilização da } \\
\text { fase física, teste indicativo de estabilidade, } \\
\text { estabilidade de pressão, dissolução intrínseca) }\end{array}$ \\
\hline
\end{tabular}


Visando aumentar a biodisponibilidade de NTZ, diferentes sistemas de veiculações devem ser estudados. Entre os diferentes sistemas de veiculação, os lipossomas são de grande interesse devido à sua biocompatibilidade, facilidade de preparação e circulação sanguínea prolongada (Allen \& Cullis, 2013). O lipossoma responsivo a estímulos, uma classe de sistemas de entrega de fármacos inteligentes, abre novos caminhos para uma liberação direcionada de moléculas de fármacos em locais tumorais específicos (De las Heras Alarcón, Pennadam, \& Alexander, 2005; Mura, Nicolas, \& Couvreur, 2013). Diferentes gatilhos (estímulos) de liberação de drogas são conhecidos e classificados de acordo com sua resposta à luz (Park, Lee, \& Kim, 2009), mudanças no pH (Zhu \& Chen, 2015), temperatura (Shang et al., 2013) e enzimas (Hu et al., 2014) etc.

As Nanopartículas Lipídicas Sólidas (NLS) são sistemas carreadores compostos por uma matriz lipídica e surfactantes. As NLSs possuem alta biodisponibilidade, protegem os fármacos de degradação, são capazes de modular a sua liberação, são biodegradáveis, possuem boa tolerabilidade e baixa toxicidade (Naseri, Valizadeh, \& Zakeri-Milani, 2015). NLS é um sistema carreador empregado quando se deseja aumentar a biodisponibilidade de fármacos pouco solúveis em água como a NTZ (Abbasalipourkabir, Fallah, Sedighi, Maghsood, \& Javid, 2016). Abbasalipourkabir e colaboradores (2016) em seu trabalho prepararam NLS contendo NTZ, caracterizaram e avaliaram a sua efetividade como sistema de liberação. No estudo, obtiveram NLS com eficiência de encapsulação de NTZ de até 91,81\% e capacidade de carga de 18,26\%. Além disso, as NLS obtidas apresentaram liberação lenta do princípio ativo, o que a caracteriza como um sistema de liberação sustentada. Concluiu-se que NLS podem ser um bom sistema carreador para liberação sustentada de NTZ.

Darwish, Bayoumi e El-Kolaly (2018) objetivando aumentar a biodistribuição de NTZ para uso em oncologia a incorporou em lipossomas. A fim de aumentar o direcionamento das nanopartículas para as células neoplásicas foi adicionada a bicamada lipídica uma ftalocianina lipofílica fotossensibilizadora e a NTZ lipossomal apresentou tamanho de 192, $2 \mathrm{~nm}$, enquanto a NTZ lazer responsiva apresentou um tamanho ainda menor de $87,4 \mathrm{~nm}$, observando que os lipossomas contendo NTZ e ftalocianina constitui um sistema carreador bem sucedido para a entrega seletiva ao tecido tumoral com uma biodistribuição superior às formulações convencionais.

Outro sistema carreador para NTZ encontrado na literatura foram as microesferas. Sachan, Gupta, Kumari e Ansari (2018) desenvolveram e avaliaram microesferas pela técnica de gelificação iônica usando como polímeros a etilcelulose e carbopol e verificaram como resultado que a melhor formulação era composto por quantidades iguais de ambos polímeros (90 mg/90 mg), $30 \mathrm{mg}$ de NTZ, 3\% de alginato de cálcio e cloreto de cálcio, causando maior eficiência de aprisionamento do fármaco $(85,50 \%)$ e porcentagem de liberação do fármaco após $10 \mathrm{~h}$ de $50,25 \%$.

Em outro estudo, Sachan e Gupta (2017) formularam nove lotes de comprimidos de duas camadas de NTZ, sendo a primeira de liberação imediata para dose de carga, preparada usando NTZ em combinação com HPMC E15 e a segunda camada de liberação sustentada como dose de manutenção usando glicolato de amido sódico como superdesintegrante, tendo a melhor formulação apresentado liberação superior do fármaco em até 12 horas em comparação com as demais formulações. Assim, os autores ressaltam que o sistema de liberação proposto é uma alternativa viável para administração sustentada de nitazoxanida e para melhorar a adesão do paciente ao tratamento.

Além dos sistemas carreadores também foram encontrados estudos de desenvolvimento de comprimidos de liberação modificada contendo NTZ. Srikala, Priya e Nadendla (2020) desenvolveram comprimidos de liberação prolongada com hidrogel superporoso. $\mathrm{O}$ uso de hidrogéis superporosos em substituição aos géis convencionais é uma estratégia utilizada para melhorar o perfil de intumescimento de comprimidos de liberação prolongada. A formulação com melhor desempenho apresentou um bom perfil de intumescimento assim como máxima taxa de liberação do fármaco, chegando a 99:52\% de liberação cumulativa. O estudo demonstrou que comprimidos com hidrogel superporoso pode ser útil quando se deseja obter uma formulação de liberação prolongada gastrorretentiva. 
Suresh et al. (2016) também desenvolveram uma formulação de comprimido de liberação prolongada testando os polímeros Eudragit L 100, Eudragit S 100 e etilcelulose. As formulações com Eudragit L 100 não apresentaram o perfil de liberação requerido que é uma liberação estendida até 12 horas e a melhor formulação obtida foi a que se empregou o Eudragit S 100 como polímero pois apresentou um melhor perfil de liberação seguindo uma cinética de liberação de ordem zero.

Soma-se a esses estudos, a geração de novas fases sólidas para transpassar a barreira da solubilidade limitada, entre eles o desenvolvimento de cocristais, como descrito por Salas-Zúñiga et al. (2019). A síntese e caracterização de cocristais NTZ já foram anteriormente relatadas, assim, o estudo referido buscar verificar comparativamente o NTZ e seus cocristais. Salas-Zúñiga et al. (2019) sintetizaram os cocristais por meio de processo de moagem líquido-assistida (LAG), resultando em ácido nitazoxanida-glutárico (NTZ-GLU, 1:1) e ácido nitazoxanida-succínico (NTZ-SUC, 2:1), por meio da moagem utilizando de solvente a acetona e realizaram experimentos, onde HPMC e Methocel ${ }^{\circledR} 60 \mathrm{HG}$ foram selecionados os melhores polímeros, pois na presença de $0,5 \%$ p / v de polímeros celulósicos pré-dissolvidos a precipitação de NTZ foi inibida, atingindo concentrações de pelo menos $1,0 \mathrm{mg} / \mathrm{mL}$.

Ademais, testes de dissolução de pó foram realizados, o qual apresentou perfis semelhantes para os cocristais e NTZ puro e, em condições "não-sink", a análise mostra que NTZ-GLU e NTZ-SUC têm propriedades de dissolução superiores em comparação com o fármaco original, sugerindo que a cinética de dissolução das fases cocristalinas é extremamente rápida. $\mathrm{O}$ estudo demonstra que a incorporação de um excipiente apropriado em concentração adequada pode ser um fator chave para induzir e manter a solubilização de fármacos fracamente solúveis a partir de formas sólidas co-cristalizadas. Desse modo, os cocristais podem ser adequados para o desenvolvimento de formas farmacêuticas sólidas com adequada biodisponibilidade e eficácia no tratamento de doenças (Salas-Zúñiga et al., 2019). Nas pesquisas de Félix-Sonda et al., 2014 cocristais com ácido succinico (NTZ- SUC), ácido glutárico (NTZ-GLU) e 2,5-di-hidroxibenzoico foram formulados buscando melhorar a solubilidade e taxas de dissolução da NTZ. Os autores verificaram que embora os co-cristais farmacêuticos NTZ-SUC e NTZGLU tenham se mostrado estáveis sob condições indicativas de estresse de estabilidade de fase (temperatura e umidade elevadas), eles demonstraram ser instáveis em soluções aquosas simulando condições gastrointestinais fisiológicas. Entretanto, as fases sólidas dos cocristais NTZ-SUC e NTZ-GLU apresentaram melhor estabilidade quando comparadas ao NTZ, achado que pode ser relevante para as especificações farmacotécnicas da molécula.

Mohan e Alur (2019) em seu estudo desenvolveu e avaliou comprimidos de matriz de liberação sustentada usando diferentes concentrações de polímeros hidrofílicos (HPMC K4M, HPMC K15M e combinação HPMC K4M, Polyplasdone XL10 e PVPK30) para verificar o efeito do polímero e aglutinante na liberação da NTZ. Como resultado encontrado pelos autores, das 12 formulações preparadas pelo método de granulação úmida, apenas a formulação 12 apresentou as melhores características estendendo a liberação do fármaco in vitro em até $12 \mathrm{~h}$. O perfil de dissolução in vitro de comprimidos de liberação sustentada de nitazoxanida indicou que os comprimidos de liberação sustentada de nitazoxanida poderiam ter um desempenho terapêutico melhor do que os comprimidos convencionais, com melhor adesão do paciente e eficácia aprimorada. Da mesma forma os autores Golamaru, Rajnarayana e Jayaveera (2016) usando celulose microcristalina, Polyplasdone XL-10, Methocel K100M Prem CR, PVPK 29/32 prepararam e caracterizaram 10 formulações de comprimidos de NTZ e com base nos resultados demonstrados no artigo 98\% de liberação in vitro ocorreu em até 12h para a formulação 9 (F9), o Methocel K100M 5\% foi selecionado como o melhor retardante de liberação. Portanto, a investigação dos autores apontou que a formulação desenvolvida foi capaz de sustentar a liberação do fármaco em comparação com os sistemas de liberação tradicionais.

Ahirrao, Rathi, Koli, Kshirsagar e Pawar (2018) formularam três lotes de comprimidos orodispersíveis de NTZ por meio da técnica de dispersão sólida a fim de aumentar a solubilidade e a taxa de dissolução do fármaco nesse sistema em comparação com a formulação comercializada, usando PEG 4000, Polaxamer 188, crospovidona, estearato de magnésio e dióxido de silício coloidal. Os lotes de formulação de dispersão sólida foram caracterizados pelo doseamento do fármaco, 
espectroscopia FTIR, DSC e perfil de dissolução do fármaco in vitro. A partir da forma cristalina de nitazoxanida convertida em estado amorfo durante a formulação da dispersão sólida a nitazoxanida oro-dispersível foi projetada usando dispersão sólida otimizada e crospovidona como superdesintegrante. Como resultados os comprimidos orodispersíveis mostraram tempo de desintegração de cinquenta e quatro segundos e liberação de droga in vitro de $87,41 \%$, enquanto que o produto disponível comercialmente liberava apenas $68 \%$ ao final de $1 \mathrm{~h}$. Sendo a formulação 3 (NTZ $300 \mathrm{mg}$, crospovidona 18 gm, MCC 113 mg, estearato de magnésio $4 \mathrm{mg}$ e dióxido de silício coloidal $5 \mathrm{mg}$ ) escolhida dentre todas pelos melhores resultados alcançados.

Compreende-se, assim que a Nitazoxanida vem sendo abordada em diferentes contextos e por diferentes veiculações, tornando-se alvo de inúmeros estudos, mesmo já sendo considerada bem estabelecida, tendo em vista que seu reposicionamento poderá agilizar sua utilização em diversas finalidades terapêuticas, potencializando sua atividade biológica, além de minimizar as dificuldades físico-químicas e biofarmacotécnicas que inviabilizam o uso da NTZ. A figura 2 mostra um breve esquema dos principais achados descritos no nosso estudo.

Figura 2. Principais achados descritos sobre sistemas de vetorização e reposicionamento da NTZ.

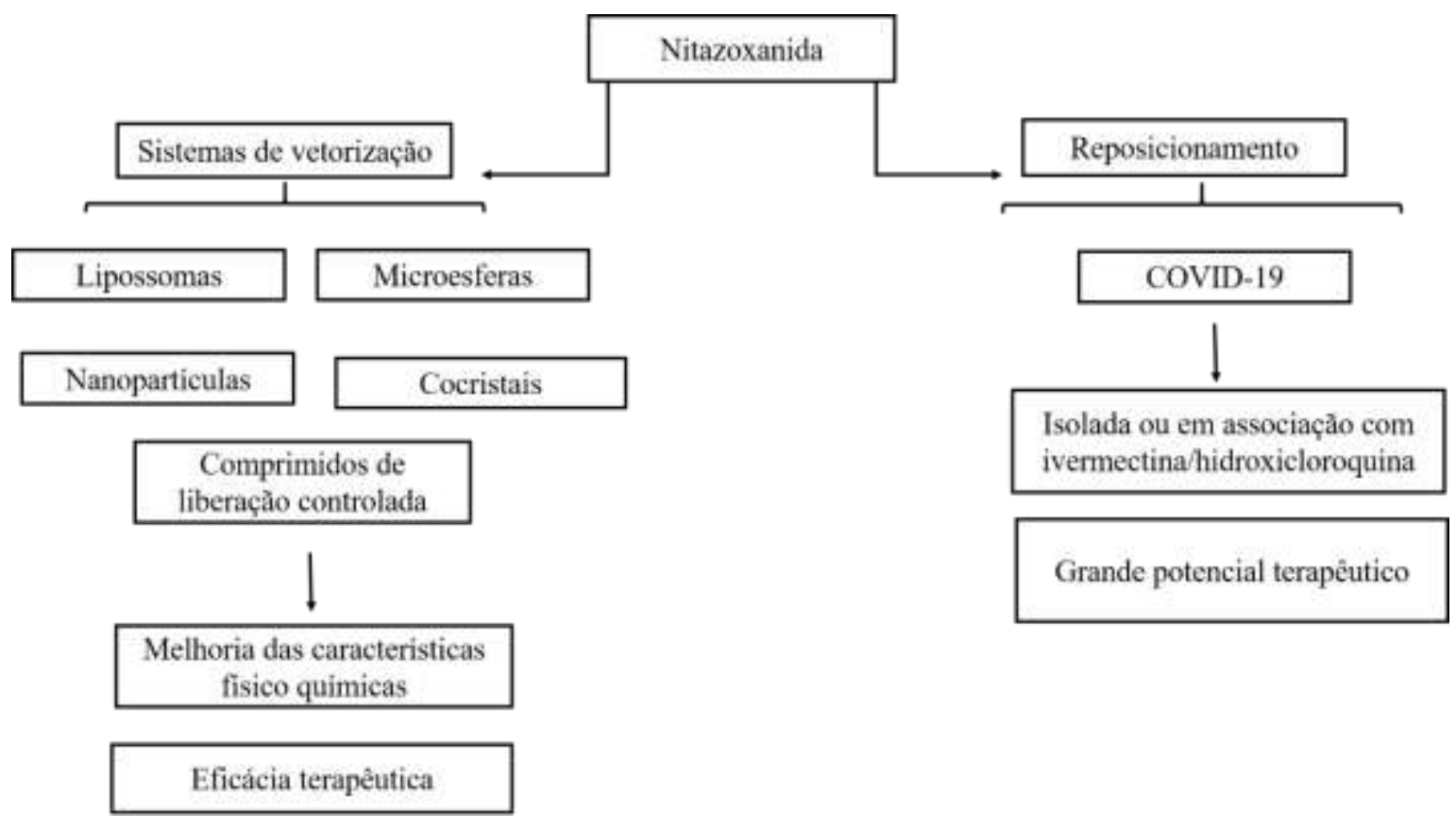

Fonte: Autores. 


\section{Conclusão}

Desse modo, observa-se que investigações acerca das ações farmacológicas e novos meios de veiculação da Nitazoxanida (NTZ) estão evoluindo, inclusive seu estudo em pacientes acometidos pela Covid-19 e em terapias associadas. Ademais, o artigo expõe estudos que abordam a importância da influência nanotecnológica e de novos sistemas de vetorização como forma de modular a liberação do fármaco, além de ultrapassar barreiras que dificultam sua biodisponibilidade, impactando nos aspectos biofarmacotécnicos da molécula e motivando seu reposicionamento para outras atividades farmacológicas que não são usadas na clínica atualmente.

Portanto, a Nitazoxanida representa um fármaco com grande potencial terapêutico e sua veiculação em novos sistemas é de suma importância, além de já serem uma realidade, tendo em vista a tecnologia que se vem investindo nos seus estudos. Apesar disso, novas investigações devem ser realizadas para o reposicionamento e a adoção na prática clínica.

\section{Referências}

Abaza, H., El-Zayadi, A. R., Kabil, S. M., \& Rizk, H. (1998). Nitazoxanide in the treatment of patients with intestinal protozoan and helminthic infections: a report on 546 patients in Egypt. Current Therapeutic Research, 59(2), pp. 116-121. DOI: https://doi.org/10.1016/S0011-393X(98)85006-6.

Abbasalipourkabir, R., Fallah, M., Sedighi, F., Maghsood, A. H., \& Javid, S. (2016). Nanocapsulation of nitazoxanide in solid lipid nanoparticles as a new drug delivery system and in vitro release study. Journal of Biological Sciences, 16(4), pp. 120-127. DOI: https://dx.doi.org/10.3923/jbs.2016.120.127.

Ahirrao, S. P., Rathi, K. S., Koli, D. B., Kshirsagar, S. J., Pawar, S. (2018). Solubility enhancement of nitazoxanide using solid dispersion. J Pharm \& Sci. v. 19 (5), 1674-1687.

Ai, N., Wood, R. D., \& Welsh, W. J. (2015). Identification of nitazoxanide as a group I metabotropic glutamate receptor negative modulator for the treatment of neuropathic pain: an in silico drug repositioning study. Pharmaceutical research, 32(8), pp. 2798-2807. DOI: https://doi.org/10.1007/s11095-015-1665-7.

Allen, T. M., \& Cullis, P. R. (2013). Liposomal drug delivery systems: from concept to clinical applications. Advanced drug delivery reviews, 65(1), pp. 36-48. DOI: https://doi.org/10.1016/j.addr.2012.09.037.

Belardo, G., Cenciarelli, O., La Frazia, S., Rossignol, J. F., \& Santoro, M. G. (2015). Synergistic effect of nitazoxanide with neuraminidase inhibitors against influenza A viruses in vitro. Antimicrobial agents and chemotherapy, 59(2), pp. 1061-1069. DOI: https://doi.org/10.1128/aac.03947-14.

Bolla, G., \& Nangia, A. (2016). Pharmaceutical cocrystals: walking the talk. Chemical communications, 52(54), pp. 8342-8360. DOI: https://doi.org/10.1039/C6CC02943D.

Botta, L., Rivara, M., Zuliani, V., \& Radi, M. (2018). Drug repurposing approaches to fight Dengue virus infection and related diseases. Front Biosci, 23, pp. 997-1019. DOI: https://doi.org/10.2741/4630.

Broekhuysen, J., Stockis, A., Lins, R. L., De Graeve, J., \& Rossignol, J. F. (2000). Nitazoxanide: pharmacokinetics and metabolism in man. International journal of clinical pharmacology and therapeutics, 38(8), pp. 387-394. DOI: https://doi.org/10.5414/cpp38387.

Chan, J. F. W., Yuan, S., Kok, K. H., To, K. K. W., Chu, H., Yang, J., ... \& Tsoi, H. W. (2020). A familial cluster of pneumonia associated with the 2019 novel coronavirus indicating person-to-person transmission: a study of a family cluster. The Lancet, 395(10223), pp. 514-523. DOI: https://doi.org/10.1016/S01406736(20)30154-9.

Darwish, W. M., Bayoumi, N. A., \& El-Kolaly, M. T. (2018). Laser-responsive liposome for selective tumor targeting of nitazoxanide nanoparticles. European Journal of Pharmaceutical Sciences, 111, pp. 526-533. DOI: https://doi.org/10.1016/j.ejps.2017.10.038.

De Carvalho, L. P. S., Lin, G., Jiang, X., \& Nathan, C. (2009). Nitazoxanide kills replicating and nonreplicating Mycobacterium tuberculosis and evades resistance. Journal of medicinal chemistry, 52(19), pp. 5789-5792. DOI: https://doi.org/10.1021/jm9010719.

De las Heras Alarcón, C., Pennadam, S., \& Alexander, C. (2005). Stimuli responsive polymers for biomedical applications. Chemical Society Reviews, 34(3), pp. 276-285. DOI: https://doi.org/10.1039/B406727D.

Dhawan, A. K., Bisherwal, K., Gandhi, V., Singal, A., \& Sharma, S. (2015). Successful treatment of cutaneous leishmaniasis with nitazoxanide. Indian Journal of Dermatology, Venereology, and Leprology, 81(6), p. 644. DOI: https://doi.org/10.4103/0378-6323.165541.

Duggirala, N. K., Perry, M. L., Almarsson, Ö., \& Zaworotko, M. J. (2016). Pharmaceutical cocrystals: along the path to improved medicines. Chemical communications, 52(4), pp. 640-655. DOI: https://doi.org/10.1039/C5CC08216A.

Elias, C. D. S. R., Silva, L. A. D., Martins, M. T. D. S. L., Ramos, N. A. P., Souza, M. D. G. G. D., \& Hipólito, R. L. (2012). When is the end?: a narrative review on the termination of the school term for mentally disabled students. SMAD. Revista eletrônica saúde mental álcool e drogas, 8(1), pp. 48-53. ISSN 1806-6976. Recuperado de https://www.semanticscholar.org/paper/When-is-the-end\%3A-a-narrative-review-on-the-of-the-EliasSilva/6a66747dea75811a472933ecc25d9a99dc28c05b 
Fan-Minogue, H., Bodapati, S., Solow-Cordero, D., Fan, A., Paulmurugan, R., Massoud, T. F., ... \& Gambhir, S. S. (2013). A c-Myc activation sensor-based high-throughput drug screening identifies an antineoplastic effect of nitazoxanide. Molecular cancer therapeutics, 12(9), pp. 1896-1905. DOI: https://doi.org/10.1158/1535-7163.mct-12-1243.

FarahatAllam, A., Shehab, A. Y., Mogahed, N. M. F. H., Farag, H. F., Elsayed, Y., \& Abd El-Latif, N. F. (2020). Effect of nitazoxanide and spiramycin metronidazole combination in acute experimental toxoplasmosis. Heliyon, 6(4). DOI: 10.1016/ j.heliyon.2020.e03661.

Félix-Sonda, B. C., Rivera-Islas, J., Herrera-Ruiz, D., Morales-Rojas, H., \& Höpfl, H. (2014). Nitazoxanide cocrystals in combination with succinic, glutaric, and 2, 5-dihydroxybenzoic acid. Crystal growth \& design, 14(3), pp. 1086-1102. DOI: https://doi.org/10.1021/cg4015916.

Gilles, H. M., \& Hoffman, P. S. (2002). Treatment of intestinal parasitic infections: a review of nitazoxanide. Trends in parasitology, 18(3), pp. 95-97. DOI: https://doi.org/10.1016/s1471-4922(01)02205-x.

Glisoni, R. J., \& Sosnik, A. (2014). Encapsulation of the antimicrobial and immunomodulator agent nitazoxanide within polymeric micelles. Journal of Nanoscience and Nanotechnology, 14(6), pp. 4670-4682. DOI: https://doi.org/10.1166/jnn.2014.8647.

Golamaru, L. R., Rajnarayana, K., \& Jayaveera, K. N. (2016). Development and in vitro-in vivo evaluation of Nitazoxanide sustained release tablets. IJPR, 6(02), p. 75. DOI: https://doi.org/10.7439/ijpr.v6i2.3017.

Guo, J., Ouyang, W., Zheng, X., Zhou, Y., Song, G., Li, H., \& Shen, Q. (2013). Preparation and toxicity test of Nitazoxanide nanoemulsion. Journal of Northwest A \& F University-Natural Science Edition, 41(1), pp. 19-24. ISSN: 1671-9387. https://jglobal.jst.go.jp/en/detail?JGLOBAL_ID=201302241750458442.

Gurgen, J., Hogan, D., Grace, E., \& Johnson, D. (2011). Nitazoxanide in the treatment of chronic cutaneous leishmaniasis resistant to traditional sodium stibogluconate. Journal of the American Academy of Dermatology, 64(1), pp. 202-203. DOI: https://doi.org/10.1016/j.jaad.2009.06.044.

Haffizulla, J., Hartman, A., Hoppers, M., Resnick, H., Samudrala, S., Ginocchio, C., ... \& US Nitazoxanide Influenza Clinical Study Group. (2014). Effect of nitazoxanide in adults and adolescents with acute uncomplicated influenza: a double-blind, randomised, placebo-controlled, phase $2 \mathrm{~b} / 3$ trial. The Lancet Infectious diseases, 14(7), pp. 609-618. DOI: https://doi.org/10.1016/s1473-3099(14)70717-0.

Harcourt, J., Tamin, A., Lu, X., Kamili, S., Sakthivel, S. K., Murray, J., ... \& Li, Y. (2020). Severe acute respiratory syndrome coronavirus 2 from patient with coronavirus disease, United States. Emerging infectious diseases, 26(6), p. 1266. DOI: https://doi.org/10.3201/eid2606.200516.

Hotez, P. J. (2014). Could nitazoxanide be added to other essential medicines for integrated neglected tropical disease control and elimination?. PLoS Negl Trop Dis, 8(3), e2758. DOI: https://doi.org/10.1371/journal.pntd.0002758.

Hong, S. K., Kim, H. J., Song, C. S., Choi, I. S., Lee, J. B., \& Park, S. Y. (2012). Nitazoxanide suppresses IL-6 production in LPS-stimulated mouse macrophages and TG-injected mice. International immunopharmacology, 13(1), pp. 23-27. DOI: https://doi.org/10.1016/j.intimp.2012.03.002.

Hu, Y., Ellis, B. L., Yiu, Y. Y., Miller, M. M., Urban, J. F., Shi, L. Z., \& Aroian, R. V. (2013). An extensive comparison of the effect of anthelmintic classes on diverse nematodes. PloS one, 8(7), e70702. DOI: https://doi.org/10.1371/journal.pone.0070702.

Jasenosky, L. D., Cadena, C., Mire, C. E., Borisevich, V., Haridas, V., Ranjbar, S., ... \& Cassell, G. H. (2019). The FDA-approved oral drug nitazoxanide amplifies host antiviral responses and inhibits Ebola virus. iScience, 19, pp. 1279-1290. DOI: https://doi.org/10.1016/j.isci.2019.07.003.

Kelleni, M. (2020). Nitazoxanide/Azithromycin combination for COVID-19: A suggested new protocol for COVID-19 early management. Pharmacological Research, 157. DOI: https://doi.org/10.1016/j.phrs.2020.104874.

Kohla, M. A., El-Said, H., El-Fert, A., Ehsan, N., Ezzat, S., \& Taha, H. (2016). Impact of nitazoxanide on sustained virologic response in Egyptian patients with chronic hepatitis C genotype 4: a double-blind placebo-controlled trial. European journal of gastroenterology \& hepatology, 28(1), pp. 42-47. DOI: https://doi.org/10.1097/meg.0000000000000492.

Laura, C., Celina, E., Sergio, S. B., Guillermo, D., Carlos, L., \& Luis, A. (2015). Combined flubendazole-nitazoxanide treatment of cystic echinococcosis: Pharmacokinetic and efficacy assessment in mice. Acta tropica, 148, pp. 89-96. DOI: https://doi.org/10.1016/j.actatropica.2015.04.019.

Lima, N. F., Picanço, G. A., Costa, T. L., Junior, R. D. S. L., \& Vinaud, M. C. (2020). In Vivo Treatment with the Combination of Nitazoxanide and Flubendazole Induces Gluconeogenesis and Protein Catabolism in Taenia crassiceps cysticerci. Acta Parasitologica. DOI: https://doi.org/10.1007/s11686-020-00263-6.

Lu, R., Zhao, X., Li, J., Niu, P., Yang, B., Wu, H., ... \& Bi, Y. (2020). Genomic characterisation and epidemiology of 2019 novel coronavirus: implications for virus origins and receptor binding. The Lancet, 395(10224), pp. 565-574. DOI: https://doi.org/10.1016/s0140-6736(20)30251-8.

Mahmoud, D. B., Shitu, Z., \& Mostafa, A. (2020). Drug repurposing of nitazoxanide: can it be an effective therapy for COVID-19?. Journal of Genetic Engineering and Biotechnology, 18(1), p. 35. DOI: https://doi.org/10.1186/s43141-020-00055-5.

Malesuik, M. D., Paim, C. S., Schapoval, E. E. S., \& Steppe, M. (2010). Development of a simple, rapid and validated spectrophotometric method for nitazoxanide in pharmaceutical formulations and comparison with HPLC. Quím. Nova: São Paulo, 33(3), pp. 739-742. DOI: https://doi.org/10.1590/S010040422010000300045

Malesuik, M. D., Gonçalves, H. M. L., Garcia, C. V., Trein, M. R., Nardi, N. B., Schapoval, E. E. S., \& Steppe, M. (2012). Identification, characterization and cytotoxicity in vitro assay of nitazoxanide major degradation product. Talanta, 93, pp. 206-211. DOI: https://doi.org/10.1016/j.talanta.2012.02.014.

Matysiak-Budnik, T., Mégraud, F., \& Heyman, M. (2002). In-vitro transfer of nitazoxanide across the intestinal epithelial barrier. Journal of pharmacy and pharmacology, 54(10), pp. 1413-1417. DOI: https://doi.org/10.1211/002235702760345518.

Mishra, R., Krishan, S., Siddiqui, A. N., Kapur, P., Khayyam, K. U., \& Sharma, M. (2020). Potential role of adjuvant drugs on efficacy of first line oral antitubercular therapy: Drug repurposing. Tuberculosis, 120, 101902. DOI: https://doi.org/10.1016/j.tube.2020.101902.

Miyamoto, Y., \& Eckmann, L. (2015). Drug development against the major diarrhea-causing parasites of the small intestine, Cryptosporidium and Giardia. Frontiers in microbiology, 6, 1208. DOI: https://doi.org/10.3389/fmicb.2015.01208. 
Mohan, A., \& Alur, A. (2019). Formulation and Evaluation of NitazoxanideSustained-Release Matrix Tablets. International Journal of Pharmaceutical and Phytopharmacological Research, 9(3), pp. 153-161. ISSN (Online) 2249-6084 (Print) 2250-1029. Recuperado de https://eijppr.com/en/article/formulation-andevaluation-of-nitazoxanide-sustained-release-matrix-tablets.

Mura, S., Nicolas, J., \& Couvreur, P. (2013). Stimuli-responsive nanocarriers for drug delivery. Nature materials, 12(11), pp. 991-1003. DOI: https://doi.org/10.1038/nmat3776.

Naseri, N., Valizadeh, H., \& Zakeri-Milani, P. (2015). Solid lipid nanoparticles and nanostructured lipid carriers: structure, preparation and application. Advanced pharmaceutical bulletin, 5(3), 305. DOI: https://dx.doi.org/10.15171\%2Fapb.2015.043.

Noronha, D. P. \& Ferreira, S. M. S. P. (2000). Revisões De Literatura. In: Campello, B. S.V. C.; Cendón, B. V.; Kremer, J. M. (Org.). Fontes De Informação Para Pesquisadores E Profissionais (p. 191). Belo Horizonte: Ufmg.

Padmanabhan, S. (2020). Potential dual therapeutic approach against SARS-CoV-2/COVID-19 with Nitazoxanide and Hydroxychloroquine. Preprint. DOI: 10.13140/RG.2.2.28124.74882.

Pal, A. K., Nandave, M., \& Kaithwas, G. (2020). Chemoprophylactic activity of nitazoxanide in experimental model of mammary gland carcinoma in rats. 3 Biotech, 10(8), pp. 1-12. DOI: 10.1007/s13205-020-02332-z.

Park, C., Lee, K., \& Kim, C. (2009). Photoresponsive cyclodextrin-covered nanocontainers and their sol-gel transition induced by molecular recognition. Angewandte Chemie, 121(7), pp. 1301-1304. DOI: https://doi.org/10.1002/anie.200803880.

Pepperrell, T., Pilkington, V., Owen, A., Wang, J., \& Hill, A. M. (2020). Review of safety and minimum pricing of nitazoxanide for potential treatment of COVID-19. Journal of Virus Eradication, 6(2), p. 52. DOI: https://dx.doi.org/10.1016\%2FS2055-6640(20)30017-0.

Rocco, P. R. M., Silva, P. L., Cruz, F. F., Junior, M. A. C. M., Tierno, P. F. G. M. M., Moura, M. A., ..., \& Lapa E Silva, J. R. (2020). Early use of nitazoxanide in mild Covid-19 disease: randomised, placebo-controlled trial. Eur Respir J. DOI: 10.1183/13993003.03725-2020.

Rossignol, J. F., \& Maisonneuve, H. (1984). Nitazoxanide in the treatment of Taenia saginata and Hymenolepis nana infections. The American journal of tropical medicine and hygiene, 33(3), pp. 511-512. DOI: https://doi.org/10.4269/ajtmh.1984.33.511.

Rossignol, J. F., \& Stachulski, A. V. (1999). Syntheses and antibacterial activities of tizoxanide, an N-(nitrothiazolyl) salicylamide, and its O-aryl glucuronide. Journal of Chemical Research, 23(1), pp. 44-45. DOI: https://doi.org/10.1039/A806676K.

Rossignol, J. F., La Frazia, S., Chiappa, L., Ciucci, A., \& Santoro, M. G. (2009). Thiazolides, a new class of anti-influenza molecules targeting viral hemagglutinin at the post-translational level. Journal of Biological Chemistry, 284(43), pp. 29798-29808. DOI: https://doi.org/10.1074/jbc.m109.029470.

Rossignol, J. F. (2014). Nitazoxanide: a first-in-class broad-spectrum antiviral agent. Antiviral research, 110, pp. 94-103. DOI: https://doi.org/10.1016/j.antiviral.2014.07.014

Rossignol, J. F. (2016). Nitazoxanide, a new drug candidate for the treatment of Middle East respiratory syndrome coronavirus. Journal of infection and public health, 9(3), pp. 227-230. DOI: https://doi.org/10.1016/j.jiph.2016.04.001.

Rother, E. T. (2007). Revisão sistemática x Revisão narrativa. Acta Paulista de Enfermagem, 20(2). https://www.scielo.br/pdf/ape/v20n2/a01v20n2.pdf. DOI: https://doi.org/10.1590/S0103-21002007000200001

Sachan, A. K., Gupta, A., Kumari, K., \& Ansari, A. (2018). Formulation and Characterization Of Microspheres Of Nitazoxanide By Chemical Crosslinking Method. Journal of Drug Delivery and Therapeutics, 8(5), pp. 190-199. DOI: 10.22270/jddt.v8i5.1850.

Sachan, A. K., \& Gupta, A. (2017). Formulation and Evaluation of Bilayer Tablets of Nitazoxanide. Der pharmacialettre, 9(7), pp. 1-9. ISSN 0975-5071. Recuperado de

Salas-Zúñiga, R., Rodríguez-Ruiz, C., Höpfl, H., Morales-Rojas, H., Sánchez-Guadarrama, O., Rodríguez-Cuamatzi, P., \& Herrera-Ruiz, D. (2020). Dissolution Advantage of Nitazoxanide Cocrystals in the Presence of Cellulosic Polymers. Pharmaceutics, 12(1), p. 23. DOI: https://doi.org/10.3390/pharmaceutics12010023.

Sallum, A. M. C., Garcia, D. M., \& Sanches, M. (2012). Dor aguda e crônica: revisão narrativa da literatura. Acta Paulista de Enfermagem, 25 (1), pp. 150-154. DOI: https://doi.org/10.1590/S0103-21002012000800023.

Shalan, S., Nasr, J. J., \& Belal, F. (2014). Determination of tizoxanide, the active metabolite of nitazoxanide, by micellar liquid chromatography using a monolithic column. Application to pharmacokinetic studies. Analytical Methods, 6(21), pp. 8682-8689. DOI: https://doi.org/10.1039/C4AY00310A.

Shang, T., Wang, C. D., Ren, L., Tian, X. H., Li, D. H., Ke, X. B., ... \& Yang, A. Q. (2013). Synthesis and characterization of NIR-responsive Au rod@ pNIPAAm-PEGMA nanogels as vehicles for delivery of photodynamic therapy agents. Nanoscale research letters, 8(1), pp. 1-8. DOI: 10.1186/1556-276X-84.

Shigyo, K., Ocheretina, O., Merveille, Y. M., Johnson, W. D., Pape, J. W., Nathan, C. F., \& Fitzgerald, D. W. (2013). Efficacy of nitazoxanide against clinical isolates of Mycobacterium tuberculosis. Antimicrobial agents and chemotherapy, 57(6), pp. 2834-2837. DOI: https://doi.org/10.1128/aac.02542-12.

Srikala, S. V., Priya, N. S., \& Nadendla, R. R. (2020). Formulation, Characterization and Antihelminthic Activity Testing of Nitazoxanide Superporous Hydrogel Tablets. Journal of Drug Delivery and Therapeutics, 10(3-s), pp. 26-36. DOI: 10.22270/jddt.v10i3-s.4130.

Somvanshi, V. S., Ellis, B. L., Hu, Y., \& Aroian, R. V. (2014). Nitazoxanide: nematicidal mode of action and drug combination studies. Molecular and biochemical parasitology, 193(1), pp. 1-8. DOI: https://doi.org/10.1016/j.molbiopara.2013.12.002. 
Suresh, C. H., Meghana, D., Beulah, K., Soujanya, M., Prathima, C., \& Sandhya, G. (2016). Design And In vitro Characterization Of Nitazoxanide Sustained Release Tablets. J Pharmacol, 6(2), pp. 4029-4034. DOI: 10.7439/ijpr.v6i2.3017.

Tilmanis, D., Van Baalen, C., Oh, D. Y., Rossignol, J. F., \& Hurt, A. C. (2017). The susceptibility of circulating human influenza viruses to tizoxanide, the active metabolite of nitazoxanide. Antiviral research, 147, pp. 142-148. DOI: 10.1016/j.antiviral.2017.10.002.

Tilmanis, D., Koszalka, P., Barr, I. G., Rossignol, J. F., Mifsud, E., \& Hurt, A. C. (2020). Host-targeted Nitazoxanide has a high barrier to resistance but does not reduce the emergence or proliferation of oseltamivir-resistant influenza viruses in vitro or in vivo when used in combination with oseltamivir. Antiviral Research, 104851. DOI: https://doi.org/10.1016/j.antiviral.2020.104851.

Wang, Y. M., Lu, J. W., Lin, C. C., Chin, Y. F., Wu, T. Y., Lin, L. I., ... \& Ho, Y. J. (2016). Antiviral activities of niclosamide and nitazoxanide against chikungunya virus entry and transmission. Antiviral research, 135, 81-90. DOI: 10.1016/j.antivira1.2016.10.003.

Wang, M., Cao, R., Zhang, L., Yang, X., Liu, J., Xu, M., ... \& Xiao, G. (2020). Remdesivir and chloroquine effectively inhibit the recently emerged novel coronavirus (2019-nCoV) in vitro. Cell research, 30(3), pp. 269-271. DOI: https://doi.org/10.1038/s41422-020-0282-0.

Weaver, S. C., \& Forrester, N. L. (2015). Chikungunya: Evolutionary history and recent epidemic spread. Antiviral research, 120, 32-39. DOI: https://doi.org/10.1016/j.antiviral.2015.04.016.

White Jr, A. C. (2004). Nitazoxanide: a new broad spectrum antiparasitic agent. Expert review of anti-infective therapy, 2(1), pp. 43-49. DOI: $10.1586 / 14787210.2 .1 .43$.

World Health Organization. (2020). WHO Coronavirus Disease (COVID-19) Dashboard. Recuperado de https://covid19.who.int/?gclid=Cj0KCQiA3YABhCnARIsAKYDH7uq-C9cugjRYq2Ha0POjSHrtPgs7wdXyfggC4CCv_T7Hhe1MeQWn1EaAqQGEALw_wcB.

Wouters, J., \& Quéré, L. (2012). Pharmaceutical Salts and Co-Crystals. The Royal Society of Chemistry: Cambridge, UK, 351-371. DOI: https://doi.org/10.1039/9781849733502.

Zhang, R., Shang, L., Jin, H., Ma, C., Wu, Y., Liu, Q., ... \& Gao, H. (2010). In vitro and in vivo antileishmanial efficacy of nitazoxanide against Leishmania donovani. Parasitology research, 107(2), pp. 475-479. DOI: https://doi.org/10.1007/s00436-010-1906-y.

Zhu, Y. J., \& Chen, F. (2015). pH-responsive drug-delivery systems. Chemistry-An Asian Journal, 10(2), pp. 284-305. DOI: https://doi.org/10.1002/asia.201402715.

Zhu, N., Zhang, D., Wang, W., Li, X., Yang, B., Song, J., ... \& Niu, P. (2020). A novel coronavirus from patients with pneumonia in China, 2019. New England Journal of Medicine, 382, pp. 727-733. DOI: 10.1056/NEJMoa2001017. 